might represent a viable and clinically meaningful strategy for achieving the best therapeutic outcome for the individual patient and to satisfy the need to optimize resources for the patient community.

\section{References}

1. Moia R, Patriarca A, Schipani M, et al. Precision medicine management of chronic lymphocytic leukemia. Cancers. 2020;12(3):642.

2. Condoluci A, Rossi D. Genetic mutations in chronic lymphocytic leukemia: impact on clinical treatment. Expert Rev Hematol. 2019;12(2):89-98.

3. Awan FT, Al-Sawaf O, Fischer K, Woyach JA. Current perspectives on therapy for chronic lymphocytic leukemia. Am Soc Clin Oncol Educ Book. 2020;40:1-10.

4. Moia R, Patriarca A, Deambrogi C, et al. An update on: molecular genetics of high-risk chronic lymphocytic leukemia. Expert Rev Hematol. 2020;13(2):109-116.

5. Tausch E, Beck P, Schlenk RF, et al. Prognostic and predictive role of gene mutations in chronic lymphocytic leukemia: results from the pivotal phase III study COMPLEMENT1. Haematologica. 2020;105(10):2440-2447.

6. Hillmen P, Robak T, Janssens A, et al. Chlorambucil plus ofatumum$a b$ versus chlorambucil alone in previously untreated patients with chronic lymphocytic leukaemia (COMPLEMENT 1): a randomised, multicentre, open-label phase 3 trial. Lancet. 2015;385(9980):18731883.

7. Arruga F, Vaisitti T, Deaglio S. The NOTCH pathway and its mutations in mature B cell malignancies. Front Oncol. 2018;8:550.

8. Stilgenbauer S, Schnaiter A, Paschka P, et al. Gene mutations and treatment outcome in chronic lymphocytic leukemia: results from the CLL8 trial. Blood. 2014;123(21):3247-3254.

9. Prica A, Crump M. Improving anti-CD20 antibody therapy: obinutuzumab in lymphoproliferative disorders. Leuk Lymphoma. 2019;60(3):573:582.

10. Estenfelder S, Tausch E, Robrecht S, et al. Gene mutations and treatment outcome in the context of chlorambucil (Clb) without or with the addition of rituximab (R) or obinutuzumab (GA-101, G) - results of an extensive analysis of the phase III study CLL11 of the German CLL Study Group. Blood. 2016;128(22):3227.
11. Wierda WG, Byrd JC, Abramson JS, et al. Chronic lymphocytic leukemia/small lymphocytic lymphoma, version 4.2020, NCCN Clinical Practice Guidelines in Oncology. J Natl Compr Canc Netw. 2020;18(2):185-217.

12. Seymour JF, Kipps TJ, Eichhorst B, et al. Venetoclax-rituximab in relapsed or refractory chronic lymphocytic leukemia. N Engl J Med. 2018;378(12):1107-1120.

13. Fischer K, Al-Sawaf O, Bahlo J, et al. Venetoclax and obinutuzumab in patients with CLL and coexisting conditions. N Engl J Med. 2019;380(23):2225-2236

14. Moreno C, Greil R, Demirkan F, Tedeschi A, et al. Ibrutinib plus obinutuzumab versus chlorambucil plus obinutuzumab in first-line treatment of chronic lymphocytic leukaemia (iLLUMINATE): a multicentre, randomised, open-label, phase 3 trial. Lancet Oncol. 2019;20(1):43-56.

15. Shanafelt TD, Wang XV, Kay NE, et al. Ibrutinib-rituximab or chemoimmunotherapy for chronic lymphocytic leukemia. N Engl Med. 2019; 381(5): 432-443.

16. Pozzo F, Bittolo T, Arruga F, et al. NOTCH1 mutations associate with low CD20 level in chronic lymphocytic leukemia: evidence for a NOTCH1 mutation-driven epigenetic dysregulation. Leukemia. 2016;30(1):182-189.

17. Bittolo T, Pozzo F, Bomben R, et al. Mutations in the $3^{\prime}$ untranslated region of NOTCH1 are associated with low CD20 expression levels chronic lymphocytic leukemia. Haematologica. 2017;102(8):e305e309.

18. Pavlasova G, Mraz M. The regulation and function of CD20: an "enigma" of B-cell biology and targeted therapy. Haematologica. 2020;105(6):1494-1506.

19. Hallek M, Cheson BD, Catovsky D, et al. iwCLL guidelines for diagnosis, indications for treatment, response assessment, and supportive management of CLL. Blood. 2018;131(25):2745-2760.

20. Diop F, Moia R, Favini C, et al. Biological and clinical implications of BIRC3 mutations in chronic lymphocytic leukemia. Haematologica. 2020; 105(2):448-456.

21. Tausch E, Schneider C, Robrecht S, et al. Prognostic and predictive impact of genetic markers in patients with CLL treated with obinutuzumab and venetoclax. Blood. 2020;135(26):2402-2412.

22. Jaramillo S, Agathangelidis A, Schneider C, et al. Prognostic impact of prevalent chronic lymphocytic leukemia stereotyped subsets: analysis within prospective clinical trials of the German CLL Study Group (GCLLSG). Haematologica. 2019 Dec 26. [Epub ahead of print]

\title{
From weakly adhesive to highly thrombogenic: the shear gradient switch
}

\author{
Yathreb Asaad and Netanel Korin \\ Faculty of Biomedical Engineering, Technion-Israel Institute of Technology, Haifa, Israel \\ E-mail: NETANEL KORIN - korin@bm.technion.ac.il
}

doi:10.3324/haematol.2020.257030

F ormation of a blood clot within an artery, is a complex process orchestrated by numerous chemical and physical factors, including: platelets, endothelium, subendothelial matrix, soluble blood proteins involved in hemostasis e.g., fibrinogen and von Willebrand factor (VWF) and blood flow. ${ }^{1}$ The pivotal role of flow characteristics in thrombosis and hemostasis has been well recognized in the field, as blood flow regulates the physical environment of the clotting process and the transport of molecules and blood cells. ${ }^{2,3}$ More specifically, in vivo and in vitro studies under constant flow highlighted wall shear rate, the spatial rate of change in velocity near the wall which affects transport and friction forces near the wall, as a key parameter controlling the thrombosis processes. ${ }^{4}$ ${ }^{7}$ Under physiological conditions, wall shear is tightly reg- ulated in the arterial vascular system. However, under pathological conditions, such as arterial stenosis, wall shear rate can increase significantly above its physiological level. ${ }^{8}$ Thus, the study of thrombosis under pathological high wall shear rates has received considerable attention and has uncovered important shear dependent processes such as platelet shear activation and VWF unfolding. ${ }^{9,10}$ However, unlike constant wall shear conditions, in stenotic sites the flow is complex and the wall shear rate changes dramatically at the flow acceleration and deceleration zones. ${ }^{11}$ Several studies have investigated platelet aggregation mechanisms under complex shear gradient to emphasize the key role of disturbed hemodynamics in thrombus cascade. ${ }^{12}$ One important study in this field was conducted by Nesbitt et al., Nature 

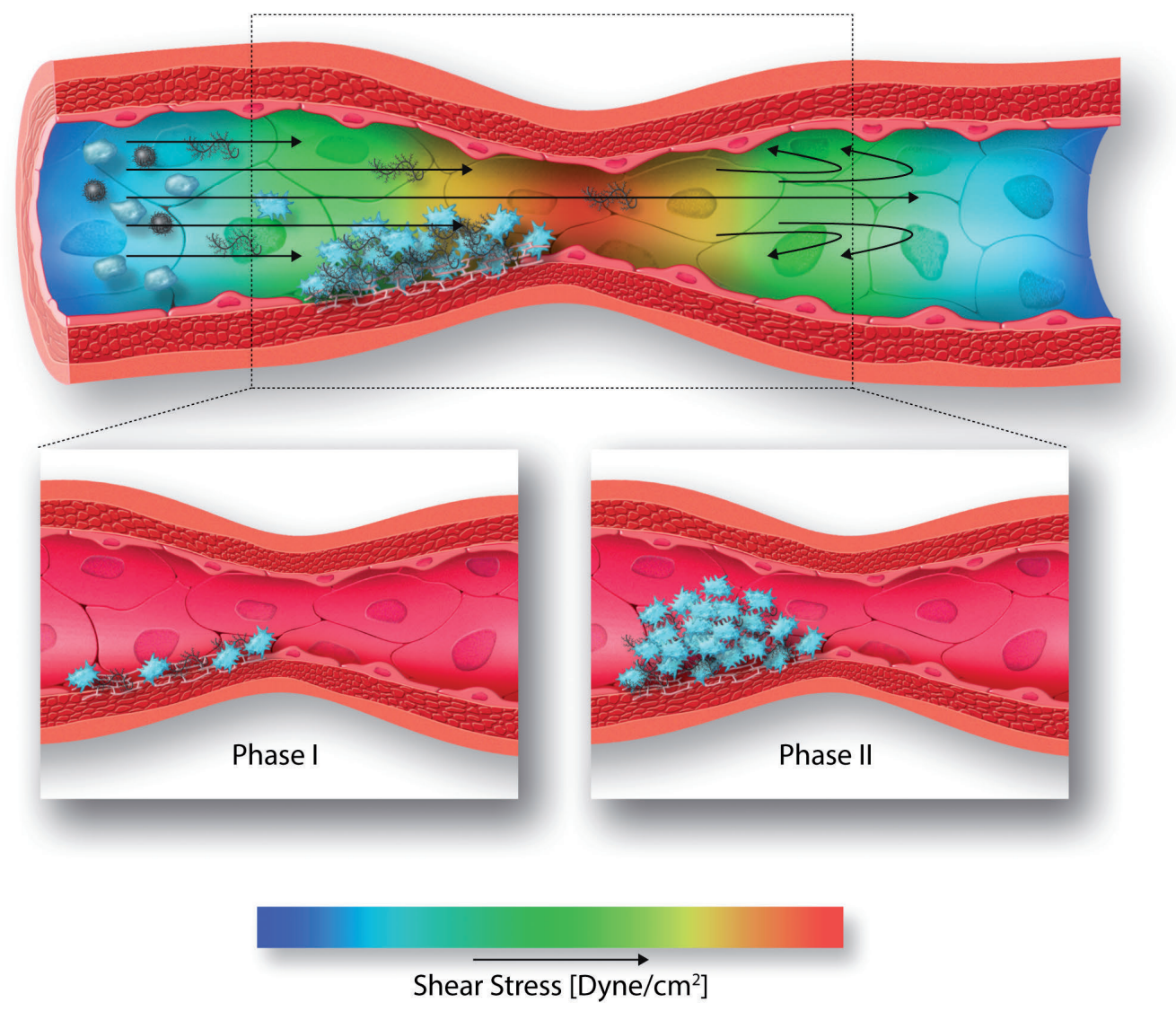

Resting Platelet

Activated Platelet

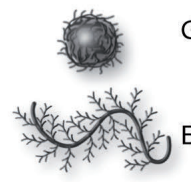

Globular VWF

Elongated VWF

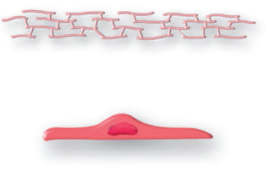

Exposed Weakly-Adhesive Protein (e.g., Fibrinogen)

Endothelial Cell
Figure1. Bi-phasic model of thrombus formation cascade at stenotic arteries under a shear gradient. (A)Wall shear rate gradually elevates within an arterial stenosis. The shear gradient alters the mechanosensitive von Willebrand factor (VWF) conformational state from globular to elongated. In atherosclerotic lesions, the extracellular matrix becomes exposed, possibly displaying weakly-adhesive proteins like fibrinogen. At these sites, thrombus is initiated when the elongated VWF adheres to the exposed fibrinogen thus creating a thrombogenic surface. Thenceforth, activated platelets from the blood stream are recruited to the vessel wall, to adhere and accumulate. (B)Thrombus formation under gradient shear is bi-phasic Phase I: individual platelets slowly adhere to VWF fibers bound to a weakly adhesive protein. Phase II: platelets rapidly aggregate and VWF elongated fibers are formed which facilitate thrombus formation.
Medicine 2009, where platelets aggregation was investigated under shear gradient hemodynamics both in vivo and in vitro. ${ }^{1}$ The study showed that local shear micro-gradients as a consequence of vessel geometry or thrombus formation, encourage the formation of platelets aggregation. ${ }^{1}$ Despite the important findings of this study, only type 1 fibrillar collagen and VWF-coated surfaces were examined, thus the possible role of other proteins, such as fibrinogen, under disturbed flow is yet to be deciphered. Thenceforth, another prominent study conducted by Westein et al., PNAS 2012, aimed to investigate stenosisdependent platelet aggregation both in vitro and in vivo. Westein et al. designed stenotic flow channels with VWF/fibrinogen-coated surfaces to represent the surface of growing thrombi and the shear rate gradient at stenotic atherosclerotic plaque sites. The combination of the physical and the biological recapitulation of the stenotic microenvironment, showed that activated platelets, VWF/fibrinogen surface combined with shear alterations at a stenosis outlet is sufficient for a strong pro-aggregatory effect. ${ }^{9}$ Despite the valuable findings of this work, it centrally highlights the role of the mechano-sensitive VWF in arterial thrombosis while less emphasis was given to the weakly-adhesive proteins role in the thrombosis cascade e.g., fibrinogen. More generally, there is still a lack of mechanistic understanding of the physical interaction between VWF and fibrinogen at stenotic sites where complex flow patterns exist.

Published in this issue of Haematologica, the study by Receveur et al., addresses this important gap of knowledge and highlights the role of weakly adhesive surfaces, e.g., fibrinogen, on thrombus formation under a shear gradient in stenotic vessels. ${ }^{13}$ Unlike most of the studies in this field, which focused on platelets behavior on an immobilized collagen or collagen/VWF surface, Receveur et al. comprehensively describe platelets adhesion mechanism under gradient shear rate on a fibrinogen surface. Surprisingly, unlike constant flow/shear conditions, under a shear gradient, weakly-adhesive protein surfaces become highly thrombogenic, see Figure 1. Moreover, the authors describe the two phases of thrombus formation under these conditions where a slow single platelet adhesion phase is followed by a rapid aggregation phase, as shown in Figure 1.

In their study, Receveur et al. used symmetric stenotic channels with a $90 \%$ lumen reduction to create a gradient 
shear stress profile with ascending shear stress values. The channels were coated with fibrinogen to simulate the early stages of thrombus formation when an extracellular matrix protein array is exposed. Whole blood with fluorescently labeled platelets was perfused over these channels while visualizing their spatial adhesion in real-time. To validate the results, platelets were perfused on straight channels that are characterized by a uniform flow regime and also on passivated channels by albumin. To validate the results in vivo, an experimental thrombosis approach was performed. An iron chloride solution and a micromanipulator were used to injure the murine carotid artery vessel wall and to create a severe stenosis which produced a shear stress gradient, respectively.

Using real-time video-microscopy, Receveur et al. succeeded in revealing several interesting observations. Under a uniform low wall shear rate, $\left(<1,000 \mathrm{~s}^{-1}\right)$, single platelets were recruited to fibrinogen-coated channel. However, when the shear rate was elevated, $\left(>1,000 \mathrm{~s}^{-1}\right)$, platelets failed to adhere. This indicates that fibrinogen has a limited affinity to its platelet ligand, integrin $\alpha \operatorname{IIb} \beta 3$, which under high wall shear rate cannot support platelets adhesion. On the other hand, when platelets were perfused in the stenotic channel, they adhered to the immobilized fibrinogen. This phenomenon was not observed, notwithstanding, when platelets experienced a similar wall shear rate in a straight channel. This unique behavior proves that platelets adhesion to immobilized fibrinogen is mediated by gradient shear due to the stenotic geometry. Another pivotal finding of this paper is the unique interaction of VWF and fibrinogen under flow. Receveur et al. show that VWF-fibrinogen interaction is shear-gradient related. In the absence of disturbed flow, the VWF-fibrinogen complex has minimum bonds to support platelets adhesion. However, when shear gradient exists, the mechano-sensitive VWF unfolds, exposes its shielded domains, and adheres to the immobilized exposed fibrinogen. Thenceforth, the sticky VWF recruits more platelets from the blood stream. This indicates that the critical step in thrombus escalation is shear gradient dependent, which forms due to a geometrical alteration at stenotic vessels.

This study also introduces a model of bi-phasic thrombus formation under shear rate gradients. The model suggests the existence of two different stages in the thrombus formation cascade: phase I and II, see Figure 1. Phase I is characterized by the slow and heterogeneous recruitment of individual platelets while phase II is characterized by long VWF sticky fibers that very rapidly contribute to platelet aggregation. More importantly, the biphasic thrombus cascade occurs only for elevated values of shear rate gradient while VWF-self association may play a crucial rule in it.

Despite the important contribution of this paper in terms of deciphering arterial thrombosis, there are some limitations. Studies have shown that parallel flow chambers and microfluidics do not give accurate results as they fail to precisely recapitulate real-scale hemodynamic forces in human arteries, important flow parameters and mass transport characteristics. ${ }^{14}$ Additionally, it is worth noting that experiments were conducted under constant flow disregarding the physiological pulsatile blood flow that exists in human arteries. Therefore, the microfluidicscale observations do not necessarily reflect the human macro-scale phenomena. Accordingly, it would be interesting to validate these observations under physiological hemodynamic conditions. Moreover, hemodynamic parameters which are highly dependent on the vessel diameter and the flow pulse, prevent us from extrapolating data from in vivo mouse experimental conditions which are different from humans not only in their biology but also in their underlying physics. ${ }^{15}$

To conclude with, this study offers a novel perspective regarding the reciprocal interactions along the axis: platelets-VWF-fibrinogen. A comprehensive outlook of these interactions along the thrombosis axis, may pave the way toward major implications for arterial thrombosis treatment and platelets adhesion down-regulation in disease conditions, which have not been examined so far. Moreover, better understanding of the processes affected by complex flow can also be valuable for the proper design of extracorporeal systems and vascular implantable devices.

\section{References}

1. Nesbitt W, Westein E, Tovar-Lopez F, et al. A shear gradient-dependent platelet aggregation mechanism drives thrombus formation. Nat Med. 2009;15(6):665-673.

2. Virchow R. Cellular pathology. As based upon physiological and pathological histology. Lecture XVI-Atheromatous affection of arteries. 1858. Nutr Rev. 1989;47(1):23-25.

3. Nesbitt WS, Mangin P, Salem HH, Jackson SP. The impact of blood rheology on the molecular and cellular events underlying arterial thrombosis. J Mol Med (Berl). 2006;84(12):989-995.

4. Holme PA, Orvim U, Hamers MJ, et al. Shear-induced platelet activation and platelet microparticle formation at blood flow conditions as in arteries with a severe stenosis. Arterioscler Thromb Vasc Biol. 1997;17(4):646-653.

5. Jackson SP. The growing complexity of platelet aggregation. Blood. 2007;109(12):5087-5095.

6. Wootton DM, Ku DN. Fluid mechanics of vascular systems, diseases, and thrombosis. Annu Rev Biomed Eng. 1999;1:299-329.

7. Bark DL Jr, Para AN, Ku DN. Correlation of thrombosis growth rate to pathological wall shear rate during platelet accumulation. Biotechnol Bioeng. 2012;109(10):2642-2650.

8. Korin N, Gounis MJ, Wakhloo AK, Ingber DE. Targeted drug delivery to flow-obstructed blood vessels using mechanically activated nanotherapeutics. JAMA Neurol. 2015;72(1):119-122.

9. Westein E, van der Meer AD, Kuijpers MJ, Frimat JP, van den Berg A, Heemskerk JW. Atherosclerotic geometries exacerbate pathological thrombus formation poststenosis in a von Willebrand factor-dependent manner. Proc Natl Acad Sci U S A. 2013;110(4):1357-1362.

10. Ruggeri ZM. The role of von Willebrand factor in thrombus formation. Thromb Res. 2007:120(Supplement 1):5-7.

11. Epshtein M, Korin N. Shear targeted drug delivery to stenotic blood vessels. J Biomech. 2017;50:217-221.

12. Jackson SP, Nesbitt WS, Westein E. Dynamics of platelet thrombus formation. J Thromb Haemost. 2009:7(Supplement 1):17-20.

13. Receveur N, Nechipurenko D, Knapp Y, et al. Shear rate gradients promote a bi-phasic thrombus formation on weak adhesive proteins, such as fibrinogen in a VWF-dependent manner [published online ahead of print, 2019 Nov 14]. Haematologica. 2020;105(10):2471 2483.

14. Khoury M, Epshtein M, Zidan H, Zukerman H, Korin N. Mapping deposition of particles in reconstructed models of human arteries. J Control Release. 2020;318:78-85.

15. Winkel LC, Hoogendoorn A, Van der Heiden K, et al. Animal models of surgically manipulated flow velocities to study shear stressinduced atherosclerosis. Atherosclerosis. 2015;241(1):100-110. 